La métaphilosophie et le problème de l'expression. Différence et interdépendance du fond et de la forme dans Qu'est-ce que la philosophie?

\title{
Anthony Bekirov
}

\section{OpenEdition}

\section{Journals}

Édition électronique

URL : http://journals.openedition.org/edl/1113

DOI : $10.4000 /$ edl. 1113

ISSN : 2296-5084

Éditeur

Université de Lausanne

\section{Édition imprimée}

Date de publication : 15 décembre 2014

Pagination : 129-136

ISBN : 978-2-940331-36-9

ISSN : 0014-2026

\section{Référence électronique}

Anthony Bekirov, «La métaphilosophie et le problème de l'expression. Différence et interdépendance du fond et de la forme dans Qu'est-ce que la philosophie ? », Études de lettres [En ligne], 4 | 2014, mis en ligne le 15 décembre 2017, consulté le 19 décembre 2020. URL : http://journals.openedition.org/edl/ 1113 ; DOI : https://doi.org/10.4000/edl.1113 


\section{LA MÉTAPHILOSOPHIE ET LE PROBLÈME DE L'EXPRESSION. DIFFÉRENCE ET INTERDÉPENDANCE DU FOND ET DE LA FORME DANS QU'EST-CE QUE LA PHILOSOPHIE?}

On entend volontiers que le dernier opus de Gilles Deleuze participe de la «métaphilosophie», entendu qu'il s'agit d'un livre de philosophie sur la philosophie. Mais bien trop souvent, on ressasse ce préfixe comme si ce à quoi il renvoyait était évident, et l'on n'interroge pas le rapport complexe et subtil qu'entretient le discours philosophique de Qu'est-ce que la philosophie? avec son sujet, relation forcément incestueuse voire schizophrène (la philosophie qui doit se séparer d'elle-même pour se prendre comme sujet). Cette tâche d'esquisser ce qui constituerait une explication du rapport entre le livre et le discours est celle à laquelle cet article s'enchaîne.

Ceci ne peut être qu'une esquisse, un essai, et non un commentaire stricto sensu. Acmé de l'œuvre deleuzien, Qu'est-ce que la philosophie? est un livre dont le titre devrait s'écrire entre guillemets, comme s'il s'agissait d'une citation. Je m'explique. Deleuze propose ici de regarder ce que les philosophes ont fait "toute leur vie» (7), ce qui est mis en jeu lorsque l'on dit philosopher. Mais ce faisant, il risquerait de tomber dans le dogmatisme: en affirmant ce qu'est la philosophie, il ne laisse pas de place à la philosophie. Disons cela autrement. Se poser cette question, de ce qu'est la philosophie, présuppose que notre point de vue doit y être extérieur; mais comment parler de ce que l'on ne connaît pas? C'est-à-dire, comment parler de philosophie si l'on n'en a pas fait? Il faut donc bien être philosophe pour parler de philosophie; mais étant philosophe, on ne peut que philosopher en voulant répondre à la question "qu'est-ce que la philosophie?", question qui suppose un point de vue extérieur à la philosophie. Deleuze est donc confronté à un paradoxe. Sa solution est la suivante: tout en voulant expliquer ce que l'on fait 
en créant des concepts, en philosophant, il doit en même temps, dans le même mouvement, se demander ce qu'il fait en se demandant et en expliquant ce qu'il fait en philosophant; dans son geste de ressaisie du geste "philosopher", il doit aussi ressaisir ce premier geste de ressaisie dans un second, ce qui lui permet de se placer hors du premier geste, qui est philosophique (un philosophe qui parle de philosophie) et conséquemment, de pouvoir tout de même se placer "à l'extérieur" afin d'en avoir l'aperçu nécessaire à la compréhension, à l'embrassade du regard (un non-philosophe qui étudie le philosophe). A la fois interne et externe à son sujet, Deleuze-le-fractal écrit donc ce qui se doit d'être nommé à juste titre une métaphilosophie.

Comment toutefois apparaît ce geste auto-réflexif second? Dans le contenu de son texte (premier geste de ressaisie), Deleuze manifeste ouvertement son dédain pour les Universaux sous le rapport de leur inclusion dans le geste du philosopher: ils sont propres à la discussion et sont aussi vides de contenu que leur forme est dogmatique ${ }^{1}$. Pourtant, le titre même de son ouvrage ne s'offusque pas de parler de «la" philosophie, et les premières pages ne s'intimident pas de la définir comme "la» connaissance par purs concepts. Notre auteur semble confirmer l'adage «faites ce que je dis, pas ce que je fais». Cependant, son emploi dogmatique des philosophèmes ne l'est qu'en apparence. Le contenu du texte démontre clairement que les Universaux ne sont que des travestis de concepts et ne rentrent pas dans le geste du philosophe (15/21); or, ce contenu, pour dire ce qu'il nous dit, se présente dans une forme qui manipule des Universaux. Ici point, entre la forme et le contenu du livre, une différence. Cette différence consiste en ceci que l'expression du contenu procède de la manière qui est récusée par le contenu. Nous

I. Une manière possible de présenter ce propos serait que les Universaux s'expriment majoritairement par le déterminant d'identité (le, la) et présupposent dans leur forme la reconnaissance de l'objet déterminé; mais les Universaux n'ayant pas de contenu particulier mais uniquement abstrait de celui-ci, ce contenu n'est assuré que par le truchement de l'hypostase, assurance qui est en même temps le témoin de l'abstraction de ce contenu et, partant, de sa vacuité: l'autonomisation d'une entité n'existant qu'en relation détache celle-ci de ses rapports nécessaires à sa compréhension. Les Universaux sont des tumeurs de la pensée, et leur forme rétive à toute détermination de contenu explique pourquoi leurs utilisateurs ne peuvent jamais s'entendre sur leur(s) définition(s). Affirmer l'existence d'un objet total sans pouvoir nonobstant en déterminer les modes, voilà qui pave la voie au théologique et donc au dogmatique (et réciproquement). 
l'avons déjà vu, ceci permet à Deleuze d'être philosophe dans le contenu et hors de la philosophie dans la forme. Mais il ne faut pas pour autant croire qu'il est externe à son sujet, la philosophie, parce qu'il en traite de manière dogmatique! Deleuze, il appert bien, n'est pas dogmatique puisqu'il sait que son exposition prend une forme dogmatique, et il nous le montre en dissociant forme et contenu dans la figure de l'opposition: il a pris conscience au préalable de ce qu'il y a de dogmatique dans la question "qu'est-ce que la philosophie» et, s'il la pose, il la médiatise à travers la connaissance du dogmatisme induit par la forme de la question qui risquerait d'amener un dogmatisme de contenu. Il se demande donc bien ce que l'on fait lorsqu'on prétend se demander ce que l'on fait en philosophant et les guillemets autour du titre mettraient en évidence le caractère de reprise réflexive de cette question déjà en soi réflexive; mais, dans sa didactique sauvage, Deleuze les omet et ne nous révèle que le "premier niveau», celui du contenu.

La puissance d'une telle démarche réside donc dans la différence constante entre ce qu'il dit et la manière dont il le dit, à condition que celle-là soit effectuée consciemment. Mais "consciemment" est un terme obscur et trompeur: en vérité, pour parler de manière pertinente du geste philosophique, la démarche de Deleuze dans son livre est nécessaire; elle se pose, que l'auteur l'ait voulu ou non, étant donné que si nous, lecteurs, pouvons prendre conscience de cette opposition entre forme et contenu, c'est parce que nous nous référons toujours d'abord au contenu dont la forme ne peut dès lors pas être indépendante, bien qu'elle n'en laisse pas d'être autonome. Si la philosophie fournit un discours (et non une discussion) en tant que contenu, la métaphilosophie de la forme produit un métadiscours, terme cependant creux s'il n'est pas expliqué plus précisément. Le contenu du livre Qu'est-ce que la philosophie? répond à la question de savoir ce que l'on (a) fait en philosophant, mais la forme répond du même coup à la question de savoir ce que l'on fait lorsqu'on essaie de répondre à la question de savoir ce que l'on fait en philosophant. Or, ce métadiscours de la forme, parce qu'il est surajouté au discours du contenu, ne peut pas se confondre avec le contenu, il n'est pas le contenu du texte (dans quel cas contraire, Deleuze ne répondrait pas à la question de départ). Entendu que Deleuze doit être interne à son sujet lorsqu'il répond à la question du livre sans guillemets, il y répond en philosophe, il fait ce qu'il dit que la philosophie fait, il manipule des concepts et en crée; mais entendu aussi que Deleuze doit être externe à 
son sujet lorsqu'il répond à la question du livre entre guillemets, il ne peut pas écrire en philosophe, il ne peut pas, dans le métadiscours de la forme, jongler avec les concepts. Et pour cause, la forme du discours est son expression. Aussi Deleuze fait-il autre chose que des concepts dans la forme du texte, il crée des affects.

La solution paraît trop simple, c'est pour cela qu'il faut la travailler au corps. Le métadiscours formel n'est pas écrit, il n'est pas dit: dans sa présentation, il n'est pas de l'ordre du $\operatorname{logos}^{2}$. Toutefois, sa mise en évidence n'est rendue possible que par la présence du logos en tant que contenu du livre par rapport auquel s'opère une différenciation. Le discours conditionne le métadiscours sans pour autant le remplir. Celui-ci ne se donne que dans l'expression du contenu, dans sa forme. Le métadiscours est donc non-philosophique, mais plus encore, il évite au discours philosophique de tomber dans le dogmatisme anti-philosophique, et par là le légitime en ceci qu'il donne au discours une assise dans le non-philosophique comme point de vue à partir duquel il est possible de le saisir et de s'observer soi-même philosophe, de s'observer créer des concepts depuis un promontoire qui ne s'est ni bâti sur, ni ne s'outille par des concepts. On pourrait alors réduire cela à la démarche scientifique. Différente est la réalité. Nous avions dit plus tôt que le discours conditionnait le métadiscours parce que nous nous rendions compte du second en renvoyant au premier sa forme; nous venons de découvrir autre chose encore, à savoir que le métadiscours permet au discours d'être philosophique et non pas dogmatique, par le fait d'être non-philosophique. Interdépendance, il y a donc bien. Maintenant, une entité non-philosophique qui ne peut pas être pensée, c'est-à-dire, ne peut pas être conceptualisée, et qui pourtant constitue le soubassement nécessaire à toute la pensée en même temps qu'elle n'aurait aucune consistance sans la pensée qui y surgit, voilà qui est la définition que donne Deleuze au plan d'immanence total (59/61-62). De nouveau le vertige. Ce qui est conceptualisé dans la matière du texte trouve son expression dans l'expression même de sa conceptualisation, y trouve son application; par le fait d'être conceptualisé selon une forme différant du contenu, le texte n'a pas besoin d'autre chose que lui-même pour prouver sa légitimité. Mais allons plus loin encore: ce métadiscours que je suis en train de

2. C'est-à-dire ne se présente pas sous la forme d'un discours articulé et intelligible, n'est pas de l'ordre du dicible. 
reconstruire, je le reconstruis par des concepts, même si ce n'est pas par eux que j'en éprouve l'existence. Il y a événement du métadiscours et pour dégager cet événement, j’ai besoin de concepts, je dois contreeffectuer l'événement du métadiscours pour remonter à ses conditions, ce que nous avons fait jusqu'ici. Mais contre-effectuer l'événement, remonter au virtuel, voilà aussi qui est la définition que Deleuze donne au geste du philosophe (150-151/160-161). Lire ce livre, comprendre la dissociation mais l'interdépendance du fond et de la forme et ce que cela implique pour le propos de Deleuze, c'est nécessairement agir en philosophe et en rester à croire que cette opposition n'est pas, c'est se maintenir dans la position stérile du commentateur. La philosophie s'adresse donc bien d'abord à des non-philosophes, parce que c'est le nonphilosophique qui sous-tend le philosophique (43-44/44-45) et partant, comprendre un texte qui n'est cohérent qu'à remettre sa consistance et sa légitimité aux mains d'une forme non-philosophique visible uniquement par un philosopher, équivaut à devenir philosophe.

Comprendre Qu'est-ce que la philosophie? émane soi-même du texte parce qu'il se reconstruit et il ne peut être reconstruit que par le lecteur. Il y a donc genèse par le lecteur du métadiscours. Et cependant, puisque ce métadiscours se pose par le jeu interne entre forme et fond, il n'a même pas besoin du lecteur pour être, puisqu'aussi longtemps qu'il n'y a pas un geste philosophique qui le contre-effectue et remonte au virtuel, il reste à l'état d'affect. J'utilise ce mot depuis tout à l'heure, mais comment en saisir les relations au livre? L'affect est ce qui donne à penser, ce qui force à penser (PS, 117), et au vu de ce que l'on vient de dire du devenir-philosophe par le lecteur face au texte de Deleuze, on pourrait rapidement en conclure que ce que manipule la forme du livre s'exprime par affects. Ce serait mettre la charrue avant les boufs. Il faut revenir au fonctionnement du métadiscours, qui est celui du plan d'immanence. Ce métadiscours, nous l'avons déjà vu, s'exprime à travers une forme via un contenu et il ne se donne d'abord que dans cette expression. Autrement dit, en lisant le fond du texte, sa forme exprimera pour nous autre chose que le simple contenu étant donné qu'il y a un écart manifeste entre l'énoncé et l'énonciation, et c'est précisément dans cet écart que se joue l'interpellation, ce qui donne à penser. Or, cette interpellation ne dépend, pour ainsi dire, que de nous qui regardons, elle advient en nous grâce à un support qui lui n'advient pas, c'est-à-dire, ne se développe pas selon des parties temporelles alors que le processus 
de reconstruction du métadiscours s'effectue dans le temps. Ce qui interpelle n'est pas soumis à la nécessité du lectorat, il n'est pas par le lectorat bien qu'il existe pour lui. Ce qui ne dépend pas du temps (ce qui n'a pas besoin d'un référent) pour être, et qui ne se donne pas sous la forme du concept (plus précisément, ce sur quoi l'on ne peut pas appliquer une détermination), voilà encore qui est un affect selon la définition de Deleuze (153-154/163-164). Et s'il force à penser, c'est parce qu'à son expression, sa forme de donation, ne co-apparait aucun concept et cela est très clair par le fait que l'énonciation ne se confond pas avec l'énoncé. Deleuze ne laisse pas ce qu'il démontre dans son livre à l'état de théorie abstraite: il en montre la réalité en l'écrivant, l'écriture fonctionne comme ce qui est écrit l'expose, à partir de quoi il sera maintenant devenu évident que l'un ne peut pas être sans l'autre. Qu'est-ce que la philosophie? éprouve le fait que plus important que la pensée, il y a ce qui donne à penser, ce dernier étant le propre de l'affect qui n'est pas alors réservé à l'œuvre artistique; un concept peut provoquer un affect et assurément, le livre de Deleuze n'est pas une œuvre d'art, mais ce qui s'en rapproche le plus. Par sa forme, il exprime le travail de l'affect sur nous, auquel correspond dans l'image de la pensée le travail de la création conceptuelle, l'effort de mettre des mots sur ce qui n'en a pas: parce qu'il en parle, Qu'est-ce que la philosophie? est une genèse de celle-ci.

Quand donc commence la philosophie? Puisqu'elle semble débuter dans l'affect, elle surgit hors du temps, elle est toujours là en attente. Certes, le texte de Deleuze, par la nécessité de l'écriture, doit exposer dans le temps ce qui n'en dépend pas, mais son tour de passe-passe, si je puis dire, est de faire "commencer" la philosophie par son livre, non pas par ce qu'il expose, mais par son exposition, par son être même qui trouve écho chez le lecteur. Il va de soi que ce n'est pas en faisant de la philosophie que l'on peut engendrer la philosophie; le nonphilosophique s'en occupera, mais alors le non-philosophique ne peut pas être le contenu du texte, qui est philosophique. En procédant ainsi, Deleuze finit d'exorciser cet objet "livre" qu'il abhorre tant ${ }^{3}$. Un problème se pose en effet à propos de la raison d'être de la publication alors que concepts et affects sont autoréférentiels, peuvent être sans le lecteur;

3. En effet, le chapitre 7 de l'ouvrage cherche, selon l'interprétation à laquelle je me rallie, à montrer l'autonomie de l'expression par rapport à ce qui la supporte, et le livre est à la philosophie ce que la toile est au tableau. 
la solution de Deleuze est de défétichiser le livre, de montrer que le livre ou le texte ne se réduit pas à l'objet, aux pages, de même que le tableau ne se réduit ni au cadre ni à la toile - «Il y a une possibilité picturale qui n’a rien à voir avec la possibilité physique...» (154-155/164) - , mais pour autant, le livre-texte ne peut pas exister sans le livre-objet au sens que nous ne pouvons notifier l'existence du premier (en dégager l'événement) sans le second, ainsi que le métadiscours ne peut être reconstruit que par le lecteur qui en notifie l'existence par la discrépance entre le fond et la forme. Publier un livre ne nous invite pas obligatoirement à lui conférer un caractère fétiche, voire sacré; temporaliser le métadiscours affectuel/ formel par une reconstruction possible découvre le livre hors du livre et nous ramène au virtuel comme le plan sur lequel toute identification n'apparaît qu'en tant qu'un des discours réalisables seulement. Qu'est-ce que la philosophie? n'est évidemment pas le seul livre à ne pas se réduire à l'objet, il est par contre le livre qui, en éclairant le fonctionnement de la création par l'auto-réflexivité, éclaire celui du livre en général et de l'image de la pensée également. Plus donc qu'un testament, cet ouvrage est celui qui porte la pensée deleuzienne à l'existence.

Anthony BeKirov Université de Lausanne 
